\title{
UniMRI: Unified Repository of Magnetic Resonance Images for Multiple Sclerosis Diagnosis
}

\author{
Wellington Silveira, Rafael Korb, Graçaliz Dimuro, Rodrigo de Bem \\ Center of Computational Sciences (C3) \\ Federal University of Rio Grande (FURG) \\ Rio Grande, Brazil \\ \{wellingtonfs, korb95, gracalizdimuro, rodrigobem\}@ furg.br
}

\begin{abstract}
Multiple sclerosis is an autoimmune disease that affects the central nervous system, destroying myelin. To detect multiple sclerosis, you need to have MRI scans so you can see the areas where myelin has been damaged. This analysis is complex and costly due to the time required to assess injuries. The use of machine learning is desirable as these exams are taken periodically. However, the number of public databases present in the literature containing patients with multiple sclerosis is small when compared to the amount of data needed to train deep neural networks. Thus, the objective of this work is to join public databases of magnetic resonance images existing in the literature, proposing a software library to manipulate and pre-process these data.
\end{abstract}

Index Terms-multiple sclerosis, magnetic resonance images, visual dataset, medical imaging

\section{INTRODUÇÃO}

A esclerose múltipla (EM) é uma doença inflamatória que acomete o sistema nervoso central, causando a destruição da bainha de mielina (desmielinização). A mielina é uma estrutura formada por uma membrana lipídica e tem como principal objetivo proteger as ligações entre as células contidas no sistema nervoso, essas ligações são conhecidas como axônios [1]. Quando a bainha de mielina é danificada, as fibras que compõem os axônios ficam expostas, comprometendo a comunicação entre as células nervosas (Figura 1). Além disso, quando essas fibras estão expostas, elas ficam vulneráveis a lesões, gerando cicatrizes nos nervos, conhecida como esclerose. Em sua pesquisa mais recente, a Federação Internacional de Esclerose Múltipla (MSIF) estimou que o número de pessoas no mundo com EM era de 2,8 milhões em 2020 [2].

Para realizar a detecção da esclerose múltipla, é necessário realizar exames de ressonância magnética para que possa ser possível visualizar as áreas onde a mielina foi danificada, esse exame é chamado de imageamento por ressonância magnética. O imageamento por ressonância magnética (RM) é uma técnica de aquisição de imagens baseada na interação entre campos magnéticos e prótons de hidrogênio existentes no corpo humano [4]. Tradicionalmente, tais imagens são usadas por um médico especialista para identificar as áreas onde a mielina foi danificada. Essa análise é complexa e custosa devido ao tempo necessário para avaliar as lesões. Além disso, a EM é uma doença neurodegenerativa que exige exames periódicos. Sendo assim, a detecção automática dessas lesões
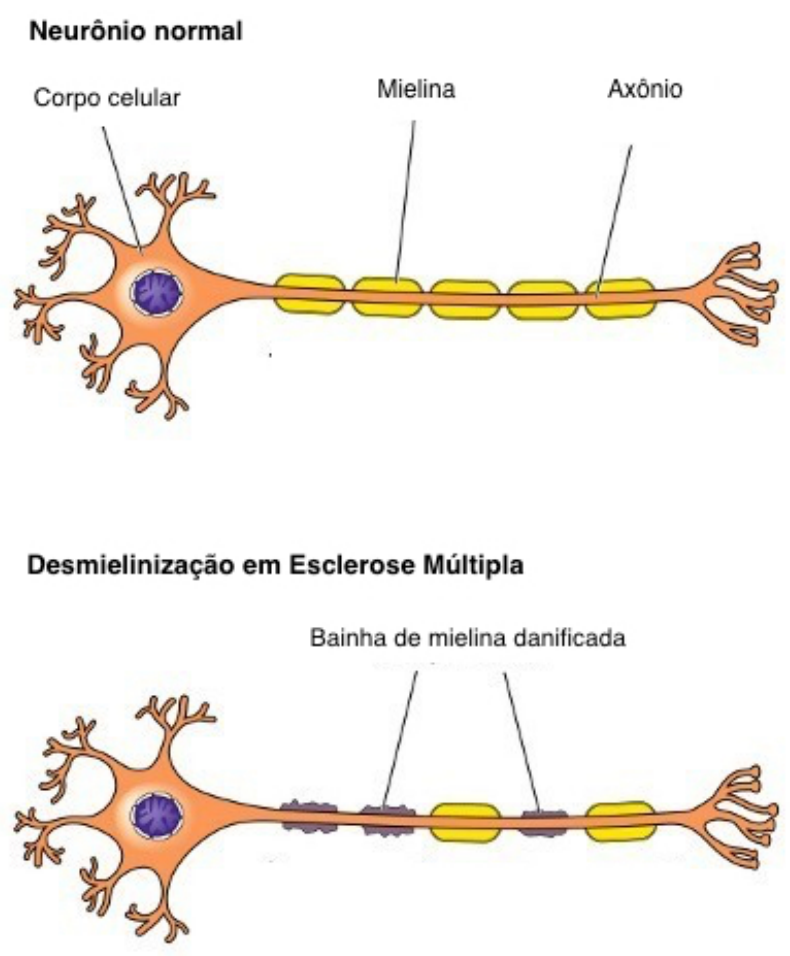

Fig. 1. Desmielinização [3].

em imagens de ressonância magnética é desejável, refletindo o tema proposto neste trabalho.

Dessa forma, o objetivo principal deste trabalho é realizar a curadoria de bancos de dados públicos de imagens de RM existentes na literatura, propondo uma biblioteca de software para fazer a manipulação, pré-processamento, adequação e integração destes bancos de dados em uma base de imagens de ressonância magnética única, contendo tanto pacientes com EM, quanto pacientes saudáveis, visando a implementação de diferentes técnicas de visão computacional e aprendizado de máquina para detecção automática de EM.

\section{TRABAlhos Relacionados}

Comumente, métodos para diagnóstico automático de EM baseados em aprendizado de máquina utilizam bancos de 
imagens de RM para treinamento e teste de seus modelos. No entanto, devido a dificuldades envolvidas na obtenção e publicização de tais imagens, muitos desse bancos de dados são mantidos privados. Tal fato dificulta reprodução de experimentos e consequentemente a comparação entre métodos propostos por diferentes grupos de pesquisa. Isso também dificulta e retarda a proposição de novos métodos de aprendizado, já que não há amplo acesso aos dados. Considerando esse contexto, fez-se uma revisão da literatura com o objetivo de encontrar bancos de dados públicos que pudessem ser utilizados para treinamento de métodos de aprendizado de máquina, e que em um segundo momento pudessem ser integrados em nosso repositório unificado. Foram selecionado bancos de imagens tanto de indivíduos com EM quanto saudáveis, diagnosticados por especialistas. Os bancos de dados encontrados na literatura são descritos a seguir.

O banco de dados OASIS-3 [5] apresenta um grande número de exames de RM (mais de 2000 sessões), contendo vários tipos de imagens, incluindo os ponderamentos T1, T2, e FLAIR. Estes ponderamentos representam variações referentes ao contraste das imagens produzidas e são discutidos na seção III. Apesar de ter como foco a Doença de Alzheimer, este repositório contém um número significativo de exames de indivíduos saudáveis, o que é relevante para o treinamento de métodos de aprendizado supervisionado de classificação mesmo no contexto da EM, já que os métodos precisam aprender a diferença entre exames normais de aqueles que apresentam doenças. Já a base de dados Multi-Modal MRI Reproducibility Resource (KIRBY) [6] contém 21 pacientes saudáveis, dispondo 42 exames de cada ponderamento FLAIR, T1 e T2. O repositório de imagens MICCAI08 [7], apresenta 51 pacientes diagnosticados com esclerose múltipla, divididos em 20 casos para treino e 31 casos para teste. O conjunto de dados conta com 51 exemplares de cada ponderamento de imagem FLAIR, T1 e T2. O conjunto de imagens MICCAI16 [8] é constituído por 53 pacientes diagnosticados com diferentes tipos de EM, onde somente 15 destes estão publicamente disponíveis, atrelados como dados de treino. Possui 15 imagens de cada ponderamento de imagem, sendo elas FLAIR, T1 (com e sem contraste), T2 e DP, além de 15 máscaras de lesão feitas por especialistas (sete segmentações manuais de lesões que resultam na máscara "consenso"). O banco de dados de Ljubljana [9] contém 30 pacientes diagnosticados com diferentes tipos de EM. São 30 imagens para cada ponderamento FLAIR, T1, T2, cada conjunto contendo 1 máscara de lesão (30 ao total). O banco de imagens ISBI 2015 [10] apresenta 19 pacientes, contendo imagem das máscaras de lesões manualmente segmentadas, 14 exames separados em treinamento e 5 para teste. $\mathrm{O}$ banco está publicamente disponível contendo imagens do tipo FLAIR, T1 e T2. Por fim, a base de dados EHEALTH [11] contém 76 exames de 38 pacientes com anotações para segmentação de lesões de EM.

Neste trabalho, todos os bancos de imagens públicos encontrados e descritos acima são reunidos em um repositórios único, o UniMRI. Em contraste com os repositórios existentes na literatura, considerados individualmente, o UniMRI contém maior quantidade de dados e por meio de um protocolo único de pré-processamento e organização (meta-dados), facilita significativamente a comparação entre diferentes métodos de aprendizado de máquina bem como o treinamento e teste de metodologias. Além disso, o nosso repositório unificado reúne exames de pacientes saudáveis bem como exames onde lesões de EM estão presentes. Esta característica também difere de outros bancos de dados, principalmente aqueles focados em segmentação de lesões, que apresentam apenas exames de pacientes com a doença, o que impede o treinamento de métodos de classificação, por exemplo, usando apenas estes bancos de imagens.

\section{Metodologia}

Os exames de imageamento por ressonância magnética possuem variações referentes ao contraste das imagens produzidas. Essas mudanças de contraste auxiliam na detecção de diferentes tecidos do corpo [12]. T1, T2 e Flair (Figura 2) são as escalas de contraste mais utilizadas para identificação de EM [13], essas escalas de contraste também são chamadas de ponderamentos. Esses três ponderamentos foram usados como base para a seleção dos bancos de dados públicos.

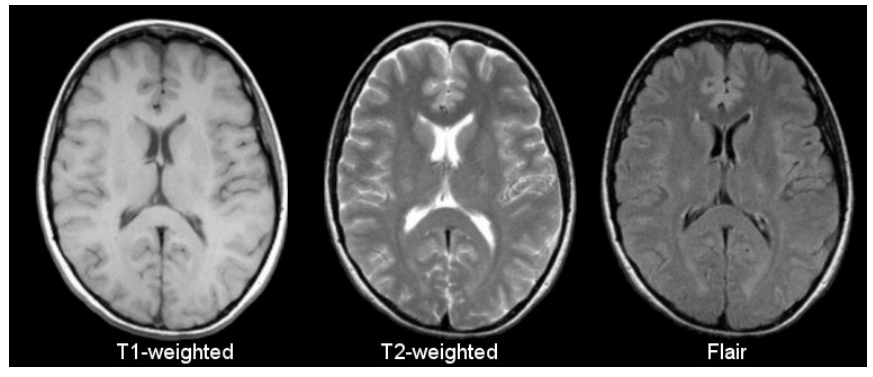

Fig. 2. Ponderamentos T1, T2 e Flair [13]

Inicialmente, foram selecionados sete bancos de dados públicos de imagens de RM contendo pacientes com EM e pacientes saudáveis. Destes bancos de dados. As informações dos bancos de dados originais são mostrados na Tabela I. Destes repositórios, foram selecionadas um total de 312 exames com imagens ponderadas em T1, T2 e Flair, sendo 191 imagens de pessoas com esclerose múltipla e 121 imagens de pessoas saudáveis. Apenas 79 exames do banco de dados OASIS 3 [5] foram utilizados para manter o balanceamento. Cada imagem presente nos bancos de dados são imagens 3D, possuindo também, com algumas exceções, informações (metadados) sobre cada exame individualmente, como: data da realização do exame, sexo do paciente, entre outros.

\section{A. Pré-processamento}

O pré-processamento dos dados é uma etapa muito utilizada por técnicas de aprendizado de máquina. Essa etapa tem como objetivo preparar, normalizar, organizar e estruturar os dados [14]. Foram utilizados cinco tipos de pré-processamento nas imagens de ressonância magnética: registro rígido, registro 
TABLE I

INFORMAÇÕES DOS BANCOS DE DADOS PÚBLICOS DE PACIENTES SAUDÁVEIS E COM ESCLEROSE MÚLTIPLA.

\begin{tabular}{|l|l|l|l|l|l|l|l|}
\hline \multirow{2}{*}{ Bancos de Dados } & \multicolumn{5}{|c|}{ Quantidade } & \multicolumn{3}{c|}{ Dados Demográficos } & \\
\cline { 2 - 8 } & Pacientes & Exames & Treinamento & Teste & Média Idade (anos) & Sexo Biológico & Diagnóstico \\
\hline OASIS 3 [5] & 1098 & 1098 & 1098 & 0 & 68,8 & $487 \mathrm{M} / 611 \mathrm{~F}$ & Saudáveis \\
KIRBY [6] & 21 & 42 & 42 & 0 & 29,0 & $11 \mathrm{M} / 10 \mathrm{~F}$ & Saudáveis \\
MICCAI08 [7] & 51 & 51 & 20 & 31 & - & - & EM \\
MICCAI16 [8] & 15 & 15 & 15 & 0 & 41,0 & $7 \mathrm{M} / 8 \mathrm{~F}$ & EM \\
LJUBLJANA [9] & 30 & 30 & 30 & 0 & 39,0 & $7 \mathrm{M} / 23 \mathrm{~F}$ & EM \\
ISBI 2015 [10] & 19 & 19 & 5 & 14 & 41,5 & $4 \mathrm{M} / 15 \mathrm{~F}$ & EM \\
EHEALTH [11] & 38 & 76 & 76 & 0 & 34,1 & $17 \mathrm{M} / 21 \mathrm{~F}$ & EM \\
\hline
\end{tabular}

afim, difusão anisotrópica, remoção do crânio e correção do campo de polarização.

1) Transformações nas Imagens: Os exames de ressonância magnética possuem diferenças de posicionamento entre si. Essas diferenças se originam tanto da máquina utilizada para a obtenção do exame, quanto do posicionamento do paciente dentro da máquina. Sendo assim, é necessário uma etapa de pré-processamento que faça o alinhamento das imagens em um único sistema de coordenadas. Esse alinhamento pode ser feito através de uma relação de mapeamento entre as imagens de RM ou através de um template [15].

Foram utilizadas duas técnicas de transformações nas imagens de ressonância magnética, o registro rígido e o registro afim. $\mathrm{O}$ registro rígido realiza transformações utilizando translações e rotações nas imagens para que fiquem todas no mesmo espaço das imagens T1 [16]. Já o registro afim utiliza um template para realizar transformações de escala e cisalhamento.

2) Remoção de Ruído e Suavização: As imagens de ressonância magnética estão sujeitas a alguns tipos de ruídos por conta das diferentes máquinas que geram essas imagens. Existem algumas técnicas para realizar a redução de ruído, como filtros gaussianos, relação da intensidade de um pixel com seus pixeis mais próximos, entre outros. Porém, essas técnicas não só suavizam o ruído, como também as bordas das imagens [17], senda elas muito relevantes na detecção de EM. Por conta disso, foi utilizado o método de difusão anisotrópica que usa equações diferenciais parciais para aumentar a eficácia da redução de ruido e preservar as bordas das imagens [18].

Além disso, a não uniformidade das intensidades de campo (bias field) é um efeito indesejável que ocorre com mais frequência em máquinas mais antigas. Embora seja sutil, algoritmos de aprendizado de máquina se tornam sensíveis a esse efeito, gerando resultados insatisfatórios [19]. Para a suavização desse efeito, foi utilizado o algoritmo $N 4$ [16], [20].

3) Remoção do Crânio: Para a detecção de esclerose múltipla, o crânio, nariz e outras partes externas da cabeça não são necessárias. Por conta disso, a remoção dessas partes é desejável, pois retira da imagem informações não úteis para o objetivo principal, que é a identificação automática de EM. Sendo assim, foi utilizado o método robust, learning-based brain extraction system (ROBEX) [21] para a remoção do crânio.

\section{RESUltados}

Neste trabalho foi possível criar uma biblioteca de software para integração de bases de dados públicas com imagens de RM rotuladas de pacientes com esclerose múltipla e saudáveis. Após isso, foram implementadas funções para fazer o pré-processamento dessas imagens, com o intuito de diminuir o ruído gerado pelos equipamentos de RM e remover informações não relevantes para a análise de lesões de EM, como o crânio e nariz. A Figura 3 mostra o resultado das transformações matriciais feitas em uma imagem de um paciente com EM (ponderamento T2).

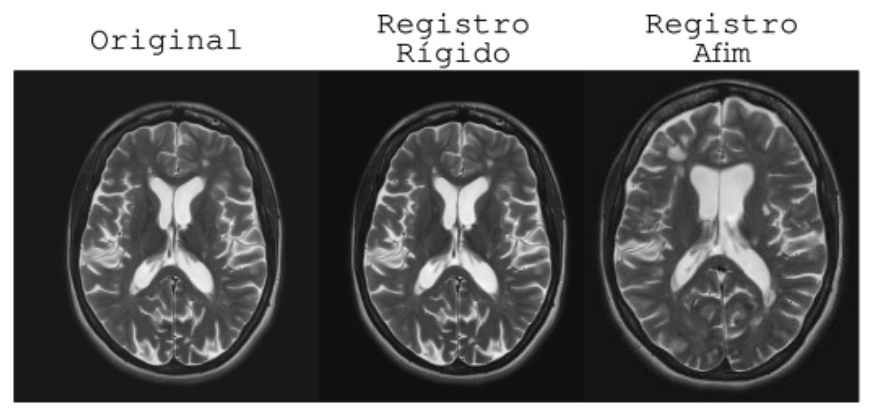

Fig. 3. Resultados das Transformações Matriciais

A Figura 4 mostra a remoção do crânio, redução de ruído e redução do bias field. Cada imagem pode ser comparada diretamente com a original, porém cada pré-processamento foi feito individualmente a partir da mesma imagem, não tendo ligação entre si. Além disso, vale salientar que a imagem que ilustra a remoção do crânio não representa a imagem original com o crânio removido, mas sim uma outra imagem do mesmo exame.

Por último, foram implementadas funções de carregamento, fatiamento (slicing) das imagens 3D em imagens $2 \mathrm{D}$ e separação dos dados em conjuntos de treinamento, validação e teste, para o uso de aprendizado de máquina para detecção automática de esclerose múltipla.

\section{A. Implementação}

A biblioteca de software foi elaborada utilizando a linguagem de programação Python 3 e uma API para armazenamento em nuvem [22] para o gerenciamento tanto dos exames 


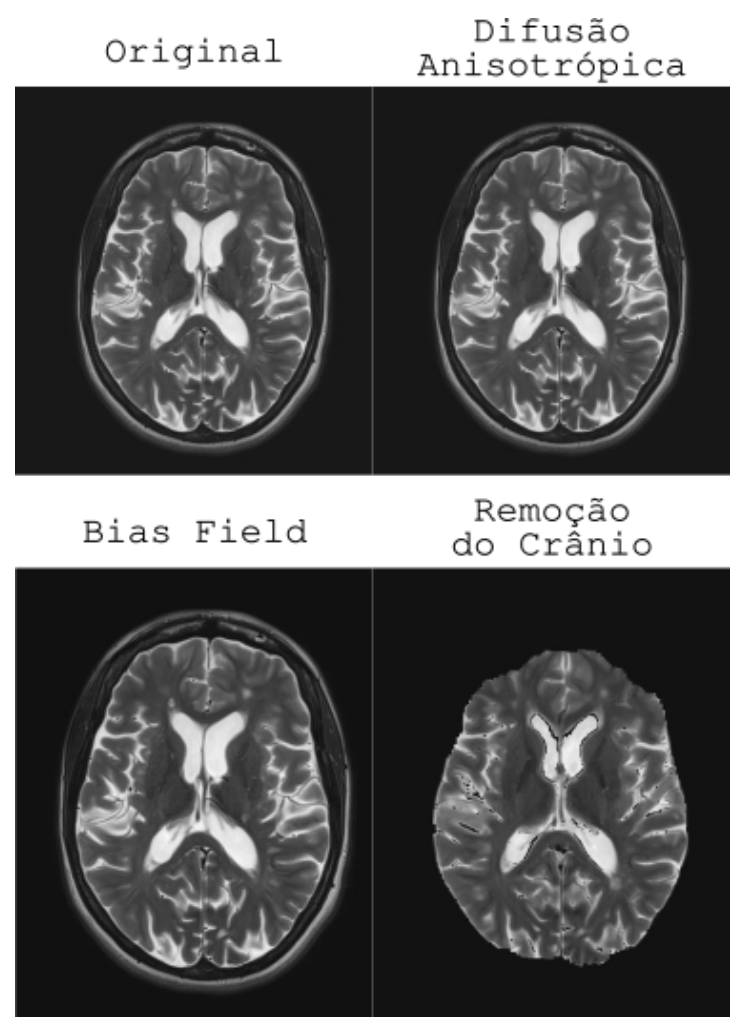

Fig. 4. Resultados da Redução de Ruído e Remoção do Crânio e Nariz

originais, como dos exames pré-processados. A biblioteca também utiliza o carregamento dos dados provenientes do framework Pytorch [23], afim de facilitar a utilização dos dados e acrescentar mais possibilidades de pré-processamentos e manipulações das imagens de RM.

Após o pré-processamento dos dados, os metadados referente a cada exame são salvos em um arquivo JSON (Figura 5) junto aos dados pré-processados, afim de que se possa ter controle sobre os detalhes de cada exame. Além disso, um outro arquivo JSON é criado contendo os diretórios e os metadados de todos os exames pré-processados, para que esses dados possam ser usados de forma simplificada por alguns outros métodos, como aprendizado de máquina, por exemplo.

$\mathrm{O}$ arquivo JSON gerado também é usado para realizar a separação dos dados de acordo com os atributos desejados. Os dados podem ser separados por sexo biológico, idade dos pacientes, saudáveis ou doentes. A separação também pode ser usada para selecionar quais bancos de dados serão usados e pré-processados.

\section{CONSIDERAÇÕES FINAIS}

Considerando o que foi exposto, o trabalho tem como principal contribuição a integração de bancos de dados públicos de imagens de RM em uma única base de dados de tamanho consideravelmente maior do que os conjuntos de dados individuais. Ressalta-se a elaboração da biblioteca de software para a manipulação e criação de tal base de dados unificada, visando a aplicação de técnicas de visão computacional e

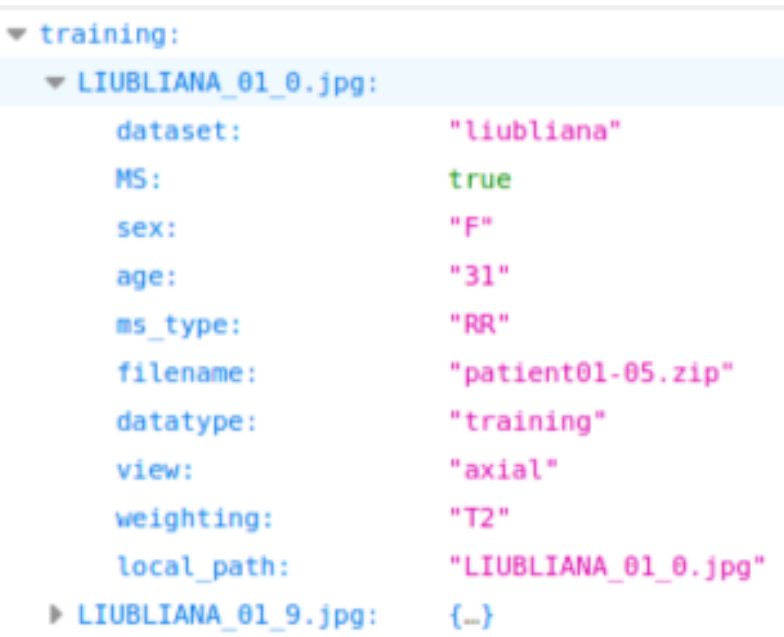

Fig. 5. Exemplo de arquivo JSON gerado pela biblioteca proposta

aprendizado de máquina para detecção automática de esclerose múltipla. Além disso, a organização e curadoria dos dados, através dos metadados reunidos no formato JSON, facilitam significativamente o uso dos dados de forma conjunta. De modo importante, é possível utilizar os metadados para facilmente fazer diferentes divisões dos dados em conjuntos de treinamento, validação e teste, em função das características dos dados, tais como idade, sexo e tipo de doença. Por fim, visando aumentar o impacto de nossa contribuição, o repositório contendo toda a implementação será disponibilizado publicamente ${ }^{1}$ para uso da comunidade científica.

\section{AGRADECIMENTOS}

Este trabalho tem apoio financeiro da FAPERGS por meio do edital ARD-21/2551-0000678-1. Wellington Ferreira da Silveira é bolsista PROBITI/FAPERGS.

\section{REFERENCES}

[1] P. B. Mendes and S. R. de Melo, "Origem e desenvolvimento da mielina no sistema nervoso central-um estudo de revisão," Saúde e Pesquisa, vol. 4, no. 1, 2011.

[2] Multiple Sclerosis International Federation - MSIF, "Atlas of ms," https://www.atlasofms.org/map/global/epidemiology/ number-of-people-with-ms, 2020.

[3] A. Teixeira, "Tudo sobre células-tronco e esclerose múltipla," http://tudosobrecelulastronco.com.br/ tudo-sobre-celulas-tronco-e-esclerose-multipla/, 2017.

[4] A. A. Mazzola, "Ressonância magnética: princípios de formação da imagem e aplicações em imagem funcional," Revista Brasileira de Física Médica, vol. 3, no. 1, pp. 117-129, 2009.

[5] P. J. LaMontagne, T. L. Benzinger, J. C. Morris, S. Keefe, R. Hornbeck, C. Xiong, E. Grant, J. Hassenstab, K. Moulder, A. Vlassenko et al., "Oasis-3: longitudinal neuroimaging, clinical, and cognitive dataset for normal aging and alzheimer disease," MedRxiv, 2019.

[6] B. A. Landman, A. J. Huang, A. Gifford, D. S. Vikram, I. A. L. Lim, J. A. Farrell, J. A. Bogovic, J. Hua, M. Chen, S. Jarso et al., "Multi-parametric neuroimaging reproducibility: a 3-t resource study," Neuroimage, vol. 54, no. 4, pp. 2854-2866, 2011.

[7] M. Styner, J. Lee, B. Chin, M. Chin, O. Commowick, H. Tran, S. Markovic-Plese, V. Jewells, and S. Warfield, "3d segmentation in the clinic: A grand challenge ii: Ms lesion segmentation," Midas Journal, vol. 2008, pp. 1-6, 2008.

${ }^{1}$ https://github.com/ReplicAI/UniMRI 
[8] O. Commowick, F. Cervenansky, and R. Ameli, "Msseg challenge proceedings: multiple sclerosis lesions segmentation challenge using a data management and processing infrastructure," in Miccai, 2016.

[9] Ž. Lesjak, A. Galimzianova, A. Koren, M. Lukin, F. Pernuš, B. Likar, and Ž. Špiclin, "A novel public mr image dataset of multiple sclerosis patients with lesion segmentations based on multi-rater consensus," Neuroinformatics, vol. 16, no. 1, pp. 51-63, 2018.

[10] A. Carass, S. Roy, A. Jog, J. L. Cuzzocreo, E. Magrath, A. Gherman, J. Button, J. Nguyen, F. Prados, C. H. Sudre et al., "Longitudinal multiple sclerosis lesion segmentation: resource and challenge," NeuroImage, vol. 148, pp. 77-102, 2017.

[11] eHealth Lab, Department of Computer Science University of Cyprus, "Mri lesion segmentation in multiple sclerosis database," http: //ehealthlab.cs.ucy.ac.cy/index.php/facilities/32-software/218-datasets, 2021.

[12] M. Kocak, "Ressonância magnética," https://www.msdmanuals. com/pt-br/profissional/t\%C3\%B3picos-especiais/princ\%C3\% ADpios-de-imagens-radiol\%C3\%B3gicas/resson\%C3\%A2ncia-magn $\%$ C3\%A9tica, 2019.

[13] D. C. Preston, "Magnetic resonance imaging (mri) of the brain and spine: Basics," https://case.edu/med/neurology/NR/MRI\%20Basics.htm, 2006.

[14] P. C. T. Gomes, "Conheça as etapas do pré-processamento de dados," https://www.datageeks.com.br/pre-processamento-de-dados/, 2019.

[15] J. E. C. Castanho and C. L. Tozzi, "Obtenção da transformação de corpo rígido em registro de imagens com deformação usando thin plate splines," Simpósio Brasileiro de Computação Gráfica e Processamento de Imagens. Anais. Caxambu, 1996.

[16] D. Tosun-Turgut, "Rigid image registration," https://cind.ucsf.edu/sites/ cind.ucsf.edu/files/wysiwyg/education/RigidRegistration.pdf, 2016.

[17] J. O. Giacomantone, "Ressonância magnética funcional com filtragem pela difusão anisotrópica robusta." Ph.D. dissertation, Universidade de São Paulo, 2005.

[18] P. Perona and J. Malik, "Scale-space and edge detection using anisotropic diffusion," IEEE Transactions on pattern analysis and machine intelligence, vol. 12, no. 7, pp. 629-639, 1990.

[19] J. Juntu, J. Sijbers, D. Van Dyck, and J. Gielen, "Bias field correction for mri images," in Computer recognition systems. Springer, 2005, pp. 543-551.

[20] J. G. Sled, A. P. Zijdenbos, and A. C. Evans, "A nonparametric method for automatic correction of intensity nonuniformity in mri data," IEEE transactions on medical imaging, vol. 17, no. 1, pp. 87-97, 1998.

[21] J. E. Iglesias, C.-Y. Liu, P. M. Thompson, and Z. Tu, "Robust brain extraction across datasets and comparison with publicly available methods," IEEE transactions on medical imaging, vol. 30, no. 9, pp. 16171634, 2011.

[22] O'Dwyer, "Api mega," https://github.com/odwyersoftware/mega.py, 2020.

[23] PyTorch, "Framework pytorch," https://pytorch.org/, 2021. 